Service social

\title{
Les difficultés psychosociales vécues par les enfants mineurs de personnes atteintes de maladie mentale
}

\author{
Marc Boily, Vivian Lew et Pauline Morissette
}

Volume 47, numéro 3-4, 1998

Enfance : enjeux et réalités 2000

URI : https://id.erudit.org/iderudit/706802ar

DOI : https://doi.org/10.7202/706802ar

Aller au sommaire du numéro

\section{Éditeur(s)}

École de service social de l'Université Laval

ISSN

1708-1734 (numérique)

Découvrir la revue

Citer cet article

Boily, M., Lew, V. \& Morissette, P. (1998). Les difficultés psychosociales vécues par les enfants mineurs de personnes atteintes de maladie mentale. Service social, 47(3-4), 247-287. https://doi.org/10.7202/706802ar
Résumé de l'article

Cet article présente les résultats d'analyse d'entrevues menées auprès d'adultes témoignant de ce qu'ils ont connu dans leur enfance en ce qui concerne leur vie avec un parent atteint de maladie mentale. "L'analyse des situations du point de vue de ceux qui les subissent " (Mayer, 1997, p. 217) nous a semblé indiquée pour cette étude et a permis de donner la parole à ceux qui vivent ces situations. Le phénomène est exploré sous deux angles : celui des situations particulières vécues par ces enfants à des phases aiguës de la maladie de leurs parents en lien avec des dimensions de la détresse psychologique; et celui du rôle parental en lien avec les dimensions de la négligence parentale et de la maltraitance. Notre objectif est de sensibiliser les intervenants et les responsables de la programmation de services à cette problématique et à l'importance d'agir auprès de ces familles. 


\section{Les difficultés psychosociales vécues par les enfants mineurs de personnes atteintes de maladie mentale}

Marc BOILY ${ }^{1}$

Viviane LEW

Pauline MORISSETTE

Cet article présente les résultats d'analyse d'entrevues menées auprès d'adultes témoignant de ce qu'ils ont connu dans leur enfance en ce qui concerne leur vie avec un parent atteint de maladie mentale. "L'analyse des situations du point de vue de ceux qui les subissent » (Mayer, 1997, p. 217) nous a semblé indiquée pour cette étude et a permis de donner la parole à ceux qui vivent ces situations. Le phénomène est exploré sous deux angles : celui des situations particulières vécues par ces enfants à des phases aiguës de la maladie de leurs parents en lien avec des dimensions de la détresse psychologique; et celui du rôle parental en lien avec les dimensions de la négligence parentale et de la maltraitance. Notre objectif est de sensibiliser les intervenants et les responsables de la programmation de services à cette problématique et à l'importance d'agir auprès de ces familles.

This article presents the results of an analysis done on interviews conducted with adults, who related their childhood experiences in living with a parent suffering from mental illness. The phenomenon is examined from two angles: the distinct situations experienced by these children, during the acute stage of their parents' mental illness, with respect to the various dimensions of the psychological distress; and that of the parental role, as it relates to the dimensions of neglect and illtreatment. Our objective is to heighten awareness of these issues in persons interceding and in program specialists, as well as to stress the importance of intervention in families concerned.

1 Nous tenons à remercier le $D^{\text {re }}$ Viviane Lew et $M^{\text {me }}$ Pauline Morissette qui ont contribué à la réalisation de cet exercice et ont accepté de signer comme coauteures. Un merci particulier va à Francine Brassard-Lamer qui a généreusement accordé de son temps à la correction. Nous remercions aussi les personnes qui ont apporté leur contribution en témoignant de ce qu'elles ont vécu. 
Les effets de la maladie mentale d'un adulte sur le bien-être de ses enfants ainsi que sur le rôle parental sont des phénomènes qui commencent à peine à intéresser certains chercheurs et cliniciens, si l'on se fie à la littérature existante en psychiatrie et en négligence parentale. Or, il est reconnu que la maladie mentale d'une personne a des effets sur son fonctionnement social et sur son réseau familial. Depuis quelques années, les recherches parlent de plus en plus clairement du fardeau familial de ces familles, mais elles restent plutôt silencieuses sur la parentalité des personnes atteintes de maladie mentale et, par conséquent, sur l'impact de la maladie mentale du parent sur la qualité de vie de ses enfants. Depuis quelques années maintenant, nous nous intéressons au processus de réadaptation psychosociale afin qu'il intègre la dimension du rôle parental de la personne souffrant de maladie mentale, de même qu'aux situations particulières vécues par les enfants mineurs dans ces familles en particulier. Cet intérêt soutenu, depuis 1992, pour ces problématiques nous a amenés à faire un mouvement d'aller-retour de la pratique à la théorie au fur et à mesure du développement de nos "connaissances " (Boily et Lew, 1997; Lew et Boily, 1999). L'objectif de cet article est de contribuer à l'émergence de connaissances en présentant les résultats d'analyse d'entrevues effectuées auprès d'adultes témoignant rétrospectivement de leur vécu pendant l'enfance auprès d'un parent atteint de maladie mentale. Notre exercice étant exploratoire, " l'analyse des situations du point de vue de ceux qui les subissent » (Mayer, 1997, p. 217) nous a semblé indiquée pour cette démarche et a permis de donner la parole à ceux qui vivent ces situations.

Nous avons choisi d'explorer le phénomène sous deux angles, soit : 1) celui des situations particulières vécues par les enfants à la phase aiguë de la maladie de leur parent, en lien avec certaines dimensions de la détresse psychologique; et 2) celui du rôle parental en lien avec les dimensions de la négligence parentale et de la maltraitance. L'objectif du présent article ${ }^{2}$ vise à sensibiliser

2 Cet article a été rédigé en 1997-1998 à la suite d'un exercice réalisé dans le cadre de notre programme de maîtrise. C'est dans une perspective de contribution aux efforts de développement des connaissances sur ce sujet et afin d'aider nos collègues cliniciens préoccupés par ce sujet que nous avons fait l'effort de rédaction du présent article. Celui-ci reflète l'état d'avancement de nos connaissances à cette époque. 
les intervenants et les responsables de la programmation de services à cette problématique et à l'importance d'agir auprès de cette clientèle. L'analyse des résultats nous amène, notamment, à suggérer des pistes d'intervention afin que le processus de réadaptation psychosociale en psychiatrie intègre la dimension du rôle parental de la personne souffrant de psychopathologie.

\section{ORIENTATIONS DES SERVICES EN SANTÉ MENTALE}

La Loi sur les services de santé et les services sociaux (loi 120), le bilan d'implantation de la Politique de santé mentale (Gouvernement du Québec, 1997a), les consultations qui ont mené par la suite au plan d'action pour la transformation des services en santé mentale constituent des déterminants qui influencent la recherche et la planification des services au Québec.

Depuis les années 1960, le traitement des personnes souffrant de maladie mentale a été influencé par le mouvement de la désinstitutionnalisation et, très récemment, par ce qu'on appelle le virage ambulatoire. Le nombre de lits pour les soins en santé mentale au Québec a été réduit considérablement dans les dernières années et cette tendance semble se poursuivre. Le nombre de lits en soins psychiatriques de courte et de longue durée est passé de 6000 qu'il était en 1995 à 4779 au 31 mars 1998, soit 0,64 lit par 1000 personnes (Gouvernement du Québec, 1998, p. 21). Le ministère de la Santé et des Services sociaux vise la norme de 0,4 lit par 1000 personnes en 2002 pour les services psychiatriques de courte et de longue durée. Ce changement se justifierait par plusieurs facteurs, comme l'amélioration des traitements (nouvelle génération de neuroleptiques), la modification des mentalités à l'égard des personnes souffrant de troubles mentaux, la mise en œuvre de pratiques considérant la personne dans son environnement, la mobilisation du réseau de soutien social (Gouvernement du Québec, 1997b), mais aussi par le fait que les coûts d'hospitalisation sont exorbitants et nécessitent le développement d'autres façons d'agir.

On note par ailleurs que la détresse psychologique de la population au Québec est un phénomène en croissance, étant passée de $14 \%$ à $26 \%$ entre 1987 et 1992 (Bellerose et collab., 1994), ce qui explique, en partie, l'augmentation du nombre de personnes 
qui reçoivent des services de santé mentale dans l'ensemble du réseau des services. Aussi, l' "Enquête québécoise sur la santé mentale des jeunes de 6 à 14 ans " menée en 1992 auprès de 2400 répondants faisait ressortir que près de $20 \%$ des enfants de ce groupe d'âge présentaient au moins une difficulté de santé mentale (Breton et collab., 1992a, p. 24). Selon les trois groupes d'âge étudiés, soit les 6-8 ans, les $9-11$ ans et les 12-14 ans, de $35,1 \%$ à $40,4 \%$ des jeunes vivent dans une famille où une personne (de la famille élargie) a déjà tenté de se suicider, s'est suicidée ou a éprouvé des problèmes de santé mentale (Breton, et collab., 1992b, p. 34). Malgré cette évolution, on hospitalise moins et les congés se donnent plus rapidement. Cette réalité a un effet sur toute la population qui doit être hospitalisée en psychiatrie. Selon nous, il est permis de croire que ces personnes risquent d'être plus fragiles et plus vulnérables au moment de leur congé de l'hôpital. La phase aiguë de la maladie se passe de plus en plus dans le milieu de vie naturel, ce qui a un effet direct sur les proches, en particulier les enfants. De plus, la maladie est reconnue comme l'une des situations qui compromettent brusquement l'équilibre du foyer. Cette réalité se manifeste de façon plus marquée lorsqu'il s'agit des difficultés d'accomplissement du rôle parental chez les personnes atteintes de maladie mentale. La parenté et les ressources de la communauté constituent des sources d'aide parfois primordiales au maintien de l'équilibre familial. À défaut d'un soutien adéquat, la maladie peut entraîner une multitude de problèmes qui menacent l'équilibre et l'intégrité familiale (Ouellette et collab., 1992). Au Québec, 8,5 \% des personnes âgées de 15 ans et plus montrent une limitation dans leurs rôles sociaux à cause de leur état de santé (Santé Québec, 1987, p. 194) et $5,3 \%$ de cette population montre une limitation à cause de troubles mentaux (Santé Québec, 1987, p. 196). Ces limites affectent plusieurs secteurs d'activité : vie professionnelle, loisirs, activités domestiques, etc. Si l'on considère les limitations dans les rôles familiaux (relation-conjoint, relation-enfants, relation-parent) qui seraient causées par l'état de santé, on note que les problèmes de santé mentale sont les principaux responsables, touchant près de $45 \%$ de la population ayant ces difficultés (Santé Québec, 1987, p. 198).

Plusieurs études mettent en évidence l'influence des problèmes de santé mentale des parents sur la santé mentale de leurs 
enfants et les risques génétiques de développer une maladie mentale si l'un des parents en est atteint. Les risques sont plus grands encore si les deux parents sont atteints. Ces études mettent aussi en perspective la vulnérabilité familiale où interagissent des facteurs génétiques et des stress environnementaux (Preziosa et collab., 1992; Inoff et collab., 1992; Hibbs et collab., 1991; Kirsh, 1989; Beardslee et collab., 1983; Orvaschel, 1983; Kaufman et collab., 1979; Anthony, 1969). Les troubles mentaux et les antécédents familiaux de troubles mentaux sont parmi les facteurs de risque importants liés à la négligence (Éthier et collab., 1994; Altemeir et collab., 1982). Plusieurs de ces recherches suggèrent que la maladie mentale du parent peut entraîner de sérieuses difficultés à évaluer les besoins de l'enfant et à y répondre. Ces comportements peuvent entraîner de la négligence ou des abus (Morisson, 1993; Ekdahl, 1962).

Les études portant sur les relations mère-enfant, surtout chez les mères schizophrènes après un épisode aigu initial, ont mis en évidence que celles-ci éprouvaient beaucoup de difficulté à répondre de manière appropriée aux besoins affectifs de leur enfant, que ce phénomène soit consécutif au retrait affectif lié à la maladie ou à la médication (Garmezy et collab., 1974; Grünebaum et collab., 1978; Rodenick et collab., 1974). Selon Weissman (1979), les personnes ayant subi un épisode dépressif aigu risquent davantage d'avoir une rechute pouvant affecter leur fonctionnement social. Cette étude montre aussi que des parents déprimés sont affectés dans leur capacité parentale. Leur degré d'implication et d'intérêt dans la vie de leurs enfants est amoindri et la communication intergénérationnelle est affectée. Les enfants ont plus de mal à parler des événements quotidiens avec leur mère, celle-ci donnant des signes de désintérêt à leur égard et manifestant un manque d'écoute. Les parents déprimés laissent voir un manque d'affection plus marqué envers leurs enfants, ce qui, en plus de l'effet négatif pour ceux-ci, engendre chez les parents des sentiments de culpabilité et d'incompétence.

II est difficile d'obtenir des données précises sur l'ampleur du phénomène. Aucune étude au Québec ne porte sur l'étendue du phénomène de la parentalité des personnes qui reçoivent des services en santé mentale. Ce type d'information ne fait pas partie des données recueillies par les établissements de santé et des 
services sociaux. Cependant, nous savons qu'entre 1993 et 1996 environ 15000 enfants ont été pris en charge annuellement par les centres jeunesse dans le cadre de la Loi sur la protection de la jeunesse (Ménard et collab., 1997). Ce nombre ne tient pas compte des enfants recevant des services d'autres organismes, tels les centres locaux de services communautaires et les hôpitaux. Selon un document de la Régie régionale de MontréalCentre, $20 \%$ des enfants de la région de Montréal faisant l'objet d'un signalement auraient un de leurs parents qui souffre de maladie mentale (Régie régionale de Montréal-Centre, 1997, p. 46). Si l'on fait un calcul rapide, on peut estimer à 3000 annuellement le nombre d'enfants québécois qui reçoivent des services de la Protection de la jeunesse et dont l'un des parents souffre de maladie mentale.

Une étude américaine menée en 1990 révèle que seulement 16 États recueillaient alors des données sur la parentalité des personnes recevant des soins en santé mentale (Nicholson et collab., 1993, p. 111). Une autre étude faite à partir de la clientèle du New York State Office of Mental Health Intensive Case Management Program révèle que, parmi les femmes de moins de 35 ans recevant des services de l'agence, $45 \%$ ont au moins un enfant de moins de 18 ans (Blanch et collab., 1993). Une autre étude américaine, menée dans l'État de New York en 1992, indique que $16 \%$ des 63000 enfants placés et $21 \%$ des 41000 enfants qui reçoivent des services de soutien en milieu familial avaient au moins un parent atteint de maladie mentale grave (Blanch et collab., 1994).

À notre avis, le manque d'information sur l'ampleur du phénomène, les difficultés particulières vécues par les parents atteints de maladie mentale, les conditions sociales et l'accomplissement de leur rôle parental reflètent une autre réalité : l'absence de services d'aide adaptés à cette population. C'est aussi un indice du peu d'intérêt que l'on accorde au rôle parental dans les services qui existent en santé mentale. Au Québec, aucun service d'aide, de soutien à la famille ou à l'enfance n'est adapté aux problèmes spécifiques de cette population, alors que le gouvernement du Québec consacre 1,2 milliard de dollars de son budget aux services en santé mentale et environ 800 millions de dollars aux services « Jeunesse et leur famille » (Gouvernement du Québec, 
1997c), soit 2 milliards de dollars au total. Au Québec, comme en Amérique du Nord, le soutien accordé aux familles aux prises avec la maladie mentale d'un proche est faible et presque absent de la planification thérapeutique. Le soutien aux familles, particulièrement lorsque la personne traitée est parent d'enfants mineurs, est totalement absent des services au Québec et peu développé aux États-Unis où il y a un manque de concertation entre les services.

Une étude nationale américaine, conduite sur une période de six mois dans les années 1990, conclut qu'au sein du milieu hospitalier ou des services publics très peu de programmes visent la réhabilitation du rôle parental des personnes atteintes de maladie mentale. À peine 9 des 69 programmes répertoriés sont centrés sur les besoins des mères ayant une maladie mentale et de leurs enfants. Seulement deux de ces programmes intègrent dans le traitement la réhabilitation du rôle parental (Nicholson et collab., 1994, p. 111).

Les enfants de personnes atteintes de maladie mentale vivent des situations particulières liées à l'hospitalisation de leurs parents. Un projet pilote (Shachnow, 1987) mené au New York HospitalCornell Medical Center propose un modèle d'intervention préventive auprès des enfants de patients hospitalisés et s'inspire des travaux de Garmezy, Grünebaum et Rodnick (1984). Cette étude souligne les phénomènes particuliers vécus par ces enfants dont l'un des parents souffrant de maladie mentale est hospitalisé. Les données recueillies à la suite du projet montrent le silence autour du phénomène. La solitude et le secret sont le lot de la plupart des enfants concernés. Très peu d'entre eux ont pu avoir recours à un substitut stable du parent au cours de l'hospitalisation ou ont eu l'occasion de discuter ouvertement de la situation de leur parent avec un proche ou un membre de l'équipe traitante du parent. Certains enfants ont eu droit à des explications mensongères ou n'ont reçu aucune explication. Pourtant, tous étaient au courant de la maladie du parent parce qu'ils avaient été témoins de crises ou parce qu'ils avaient été informés de manière indirecte. II ressort aussi de cette étude que le soutien professionnel, malgré l'évidence des besoins, était rare. Tous les enfants qui ont participé au projet ont eu une réaction de stress marquée: forte anxiété, angoisse de séparation, culpabilité, 
peurs, ambivalence à l'égard du parent malade, embarras face aux pairs et inquiétude quant à leur propre santé mentale.

\section{MÉTHOdOLOGIE}

Notre principal objectif dans cet exercice consiste à documenter le phénomène de l'exercice de la parentalité dans les familles où l'un des parents souffre de maladie mentale. Comme il s'agit d'un sujet très large, nous avons circonscrit l'objet d'étude. Dans le cadre de cet exercice, nous avons cherché à documenter la perception des personnes ayant vécu avec un parent souffrant de maladie mentale - en particulier lors de phases aiguës de la maladie -, quant aux situations reliées à l'accomplissement du rôle parental. Ces situations sont analysées sous deux angles, soit: 1) celui des situations particulières vécues par les enfants à la phase aiguë de la maladie de leurs parents, en lien avec certaines dimensions de la détresse psychologique; et 2) celui du rôle parental en lien avec les dimensions de la négligence parentale et de la maltraitance.

Afin de répondre à nos préoccupations, nous nous sommes inspirés de l'approche écologique qui permet la compréhension de situations humaines en tenant compte de plusieurs facteurs interreliés. Comme nos objectifs sont de sensibiliser tant les intervenants que les responsables de la programmation de services à cette problématique et à l'importance d'agir auprès de cette clientèle, nous avons tenté de démontrer que ces enfants et ces parents demandent une attention particulière.

Cette démonstration requiert un certain "défrichage avant de dégager les éléments prioritaires, d'en saisir la complexité et d'en faire émerger des hypothèses » (Guberman et collab., 1993, p. 230). Aussi avons nous retenu une méthode qualitative d'analyse de contenu d'entrevues dans un objectif descriptif.

Cette méthode est davantage utile dans une étude de phénomènes peu explorés et favorise l'émergence des connaissances à partir de "l'objet d'étude ». Elle a cependant le désavantage d'être plus difficilement vérifiable et généralisable et le contenu du texte est alourdi par la citation des nombreux témoignages qui donnent un sens aux propos descriptifs ou explicatifs. 
Nous avons choisi de tenir des entrevues semi-dirigées avec des personnes qui ont vécu avec un parent souffrant de maladie mentale et qui sont maintenant adultes. Ce choix nous permettait d'obtenir des témoignages de personnes qui, n'étant plus dans une situation de vulnérabilité potentielle, étaient ainsi capables de poser un regard critique sur leurs expériences d'enfant. De plus, la perception des personnes ayant vécu avec un parent souffrant de maladie mentale nous apparaissait plus pertinente que celle que nous aurions obtenue en interrogeant directement des parents souffrant de maladie mentale sur l'exercice de leur rôle. Enfin, nous avons également écarté la tenue d'entrevues avec des enfants mineurs vivant (in vivo) des situations difficiles, puisque ce choix aurait posé un problème éthique important et des difficultés à obtenir l'autorisation du parent, lui-même en besoin d'aide.

Ainsi, notre analyse des situations vécues dans l'enfance par ces personnes s'inspire d'une des dimensions de l'écologie humaine, "l'analyse des situations du point de vue de ceux qui les subissent » (Mayer, 1997, p. 217). Le nombre d'entrevues se limite à neuf $(n=9)$. La taille restreinte de cet échantillon invite à une grande prudence quant à la lecture des résultats et ne permet certainement pas la généralisation de ceux-ci. Nous avons utilisé une méthode non probabiliste, soit l'échantillon accidentel (Mayer et collab., 1991, p. 387).

Les répondants sont des volontaires recrutés parmi des personnes ayant vécu avec un parent souffrant de maladie mentale et suivi à la Clinique Mercier-Ouest du Centre hospitalier Louis-H. Lafontaine à Montréal-Est $(n=3)$, ou parmi des personnes ellesmêmes suivies à la clinique et dont un parent avait une maladie mentale $(n=6)$. Cette dernière catégorie est la plus nombreuse, car ce sont d'abord ces personnes qui se sont montrées intéressées à contribuer à l'étude dans le but d'aider d'autres enfants. Malgré le biais que pouvaient apporter ces derniers dans le corpus de données, nous avons considéré que la richesse de contenu valait le risque de la critique méthodologique.

Nous avons intégré dans notre échantillon une fratrie d'enfants mineurs n'étant plus en situation de vulnérabilité et également un couple avec des enfants mineurs dont l'un des conjoints souffre de maladie mentale. Cet échantillon permet d'obtenir des 
témoignages pour lesquels l'effort de rétrospective est moins grand, de trianguler les informations et de "bonifier" la codification et la catégorisation de thématiques. Ces neuf entrevues représentent donc 11 personnes interrogées. Ces personnes ont accepté de participer à une entrevue semi-dirigée d'une durée d'environ 45 minutes.

Malgré le nombre limité d'entrevues (9) tenues auprès de personnes témoignant de ce qu'elles ont vécu, il est intéressant de noter que, si l'on inclut la fratrie, les histoires décrites concernent 24 enfants. Nous faisons cette remarque simplement pour donner une idée de l'ampleur des témoignages recueillis, sans toutefois retenir ce dernier chiffre dans l'analyse des résultats.

Un autre élément à considérer est le fait que parmi les personnes interrogées plusieurs (six sur neuf) ont connu un problème de santé mentale. Parmi elles, cinq ont des enfants mineurs.

L'échantillon compte six femmes et cinq hommes d'âges divers: 10 ans $(n=1) ; 16$ ans $(n=1) ; 29$ ans $(n=1)$; de 34 à 42 ans $(n=7)$; et 50 ans $(n=1)$. Les entrevues ont été enregistrées sous mode audiovisuel après obtention des consentements habituels et dans le respect des règles d'éthique. Les comptes rendus in extenso des entrevues ont été dactylographiés afin de faciliter l'analyse. Le schéma d'entrevue concerne les thèmes suivants : maladie du parent, conditions sociales, rôle parental et soutien reçu.

L'analyse d'entrevue s'est déroulée en plusieurs étapes selon les méthodes suggérées par Mayer et Ouellet (1991). À l'étape 1, une première lecture flottante nous a permis d'effectuer une première catégorisation (Mayer et Ouellet, 1991, p. 484). À l'étape 2, des lectures théoriques ont été reprises afin de faire le lien avec les dimensions retrouvées dans la littérature. À cette étape, nous avons dégagé certaines similitudes entre ce qui ressortait dans cette première codification et les phénomènes décrits dans la littérature sur le thème de la détresse psychologique ainsi que sur le thème de la négligence parentale. Bien que les concepts utilisés dans les études diffèrent quelque peu sur le plan de la terminologie, nous avons procédé à l'harmonisation des concepts lorsque la signification semblait identique afin de nous assurer de l' « univocité » de ceux-ci. 
L'étape 3 vise la codification de quelques entrevues. Elle suppose des étapes préalables de catégorisation et de codification. Elle vise à produire une codification cadre qui s'inspire du modèle mixte permettant l'exploitation du matériel (Mayer et Ouellet, 1991, p. 486). L'analyse apparaît donc comme le reflet de cette codification cadre et représente l'étape 4 de la codification et de la catégorisation du corpus de données. Les prénoms ont été changés afin de préserver le caractère d'anonymat.

\section{ANALYSE DES RÉSULTATS}

L'analyse des résultats s'articule principalement autour de deux angles, soit: 1) celui des situations particulières vécues par les enfants à la phase aiguë de la maladie de leurs parents, en lien avec certaines dimensions de la détresse psychologique; et 2) celui du rôle parental en lien avec les dimensions de la négligence parentale et de la maltraitance.

Nous avons, pour cette partie, porté un regard sur les conditions sociales vécues par ces familles afin de les mettre en relief avec les facteurs de risque reconnus dans les phénomènes de la santé mentale et de la négligence parentale.

\section{DÉTRESSE PSYCHOLOGIQUE VÉCUE PAR LES ENFANTS}

Dans le cadre de l'enquête menée en 1987 sur la santé des Québécois, la détresse psychologique a été estimée dans la population des 15 ans et plus à l'aide d'une échelle de santé mentale, le Psychiatric Symptom Index (PSI), comportant 29 questions. L'instrument validé a été traduit en français et adapté à la population québécoise par Santé Québec. L'échelle de détresse psychologique est associée aux états dépressifs et anxieux, à certains symptômes d'agressivité et à des troubles cognitifs. Les résultats d'enquête montrent une augmentation de la détresse psychologique de la population. Cependant, ces recherches épidémiologiques ont des limites importantes quant aux définitions des construits des concepts, lesquelles n'ont pas toujours de valeur sur le plan du diagnostic clinique.

Dans certaines recherches, des examens cliniques non structurés sont employés pour poser un diagnostic, alors 
qu'on utilise ailleurs des entrevues cliniques structurées : d'autres études se servent de questionnaires évaluant des dimensions ou encore des mesures de dysphorie décrites à partir d'une entrevue (Seifer, 1996, p. 10).

Dans l'exercice que nous avons fait, toutes les personnes interrogées ont, selon leur perception, vécu des situations pouvant laisser croire à une certaine détresse psychologique au sens de la définition qu'en font Tousignant et Kovess (1985). Les échelles de santé mentale donnent une idée imprécise de la prévalence des troubles mentaux, mais décrivent assez bien la détresse psychologique. Les personnes que nous avons interrogées nous ont parlé de plusieurs situations qu'elles ont vécues. Nous avons regroupé ces phénomènes en tentant de les comparer aux variables contenues dans l'échelle de détresse psychologique sous les sentiments de peur ou de crainte, de tristesse, de solitude, de découragement, de désespoir et de comportement d'évitement, faisant partie des indicateurs de l'instrument. Ces situations sont liées à la maladie de leur parent. Bien que l'Index de symptômes psychiatriques soit conçu pour des personnes de 15 ans et plus et s'applique dans un contexte d'enquête, ces indicateurs ont permis d'organiser notre matériel. Toutes les personnes interrogées ont vécu, à des degrés divers et à certains moments de leur enfance, des situations correspondant à certains des indicateurs de détresse psychologique retenus. Pour certains, ces événements ont été ponctuels, mais rarement isolés. Cependant, pour la majorité des personnes interrogées, il s'agit de situations prolongées, diverses et répétitives dans leur vie. Dans certains cas, on observe aussi une gradation de la détérioration de la situation familiale qui entraîne une plus grande détresse psychologique de l'enfant.

Toutes les personnes interrogées ont vécu, dans leur enfance, des sentiments de peur ou de crainte se manifestant particulièrement lors de la décompensation du parent. Certains craignent ces moments de rechute qui peuvent avoir des conséquences catastrophiques, allant même jusqu'à s'inquiéter pour la vie du parent ou pour leur propre vie. Ils intègrent qu'ils doivent éviter tout comportement qui risque d'augmenter le stress du parent ou de le contrarier; ce qui se traduit chez certains par l'adoption de comportements de très grande docilité. 
Cécile : J'ai vécu dans la peur. T'es fine, pas parce que t'es fine, t'es fine parce que t'as peur. [...] Moi, j'avais toujours la peur de me faire tuer, la peur qu'elle tue mon petit frère. C'étaient des peurs terribles... T'es paralysée par la peur.

Peur aussi teintée de désespoir, lorsqu'ils constatent l'état parfois très détérioré de leur parent, craignant le pire et ressentant de l'impuissance devant le déni du parent quant à son état ou le refus de recevoir de l'aide.

Nathalie: Avant qu'elle rentre à l'hôpital... elle est déménagée souvent. En dernier, elle était dans un soussol où il faisait noir. Elle était encore plus maigre, elle ne mangeait plus, elle avait de la misère à se tenir debout, elle était faible. Là j'ai eu vraiment peur, j'ai dit :" $A$ va mourir, faut faire de quoi, a va mourir. " J'appelais mon grand-père : " Faites de quoi, sauvez-la... Faut la rentrer à l'hôpital, rentrez-la de force à l'hôpital. " "Ben on peut pas faire ça, faut qu'a consente à y aller. »

Peur aussi causée par le fait d'être témoin de crises psychotiques ou dépressives qui peuvent être inquiétantes pour le parent malade, ses enfants et sa famille. Les enfants assistent à des crises devant lesquelles ils se sentent impuissants ou ils ont peur de comportements qui, souvent, précèdent une hospitalisation en psychiatrie. Ils constatent que le parent ne fait plus rien, ne parle plus à personne, reste couché pendant des semaines, a des comportements plus dérangeants, comme des hallucinations auditives, visuelles.

André : Ma mère voyait des personnes qui étaient pas là. Elle entendait des cris. Elle bougeait beaucoup comme si elle pouvait pas rester à une place. Quand elle est devenue malade elle ne pouvait plus vraiment s'occuper de moi... ça a duré un bon 3-4 mois avant que maman rentre à l'hôpital.

Lors de visites à l'hôpital, l'observation du comportement d'autres personnes hospitalisées suscite aussi la crainte, l'enfant appréhendant que le parent devienne comme elles.

Nathalie: Ça me faisait peur de venir dans un hôpital psychiatrique voir ma mère. Je voyais du monde bizarre des fois, j'me disais "Ma mère a viendra pas comme 
ça? " Je trouvais ça malheureux pour elle parce que je trouvais que c'était renfermé... Y a pas beaucoup d'air et elle pouvait pas sortir.

D'autres craignent pour leur propre santé mentale et appréhendent les risques génétiques.

Nicolas: Moi, ma grosse peur, c'est de devenir comme mon père. Tu sais, j'ai vu ce que ça fait sa maladie dans ma famille et pour lui dans sa vie.

Bien que la période de décompensation soit crainte par tous, l'hospitalisation du parent est aussi source de répit et l'enfant se sent habituellement rassuré.

Cécile : Quand elle a été hospitalisée, c'était un cadeau pour moi. C'était comme une délivrance.

Le sentiment de tristesse est vécu à des intensités diverses et tire son origine de plusieurs sources. Ce qui est ressenti de façon plus aiguë, c'est le sentiment de tristesse lié à une impression d'impuissance devant la détérioration de la santé mentale et devant l'incapacité du parent à «fonctionner » psychologiquement et socialement. La tristesse apparaît encore plus présente chez l'enfant lorsque celui-ci doit renoncer à assumer les rôles de maintien de l'unité familiale ou encore de rôle parental inversé parce qu'ils sont trop lourds pour lui. Cette tristesse est alors amplifiée par des sentiments d'incompétence, de culpabilité et de trahison envers la famille et le parent malade quant au mandat implicite de maintenir la famille unie. Certains enfants semblent intégrer l'idée qu'ils ont abandonné le parent et la famille, ce qui peut détériorer l'estime de soi.

Nathalie : Je trouvais que j'avais beaucoup de responsabilités... C'est moi qui étais plus la mère. Je sentais qu'il fallait que je fasse de quoi. J'étais en sixième année... Là y a une travailleuse sociale qui est venue nous voir (à l'école) pour faire des démarches pour qu'on s'en aille de chez nous... Ça m'a fait beaucoup de peine. Mon père était parti (suicide)... Ça a fait beaucoup de peine à ma mère, et moi j'avais de la peine de lui faire de la peine. Dans le fond je pensais qu'elle penserait que je l'aimerais plus... Me semble que j'aurais dû rester chez moi et essayer de la soigner moi-même, pas aller en 
famille d'accueil, je me disais : "Ma mère va penser que je l'aime plus $»$.

Sentiment de tristesse parfois causé par des événements catastrophiques, comme le suicide du parent malade, et qui a des conséquences à long terme sur le plan du sentiment d'abandon.

Nathalie : La soirée où mon père est mort. C'était l'enfer. On est partis se coucher, mon père est venu me voir, $y$ m'a dit : " Je veux que tu sois sage avec ta mère, que tu prennes soin de toi, que tu continues tes études, pis je t'aime beaucoup quoi qu'y arrive. " Là y m'a donné un bec, y m'a donné une piasse ronde, pis y est parti... (Dans la nuit) mon père a tiré sur ma mère, après, mon père y s'est suicidé, y s'est tiré... Ça été des semaines où j'étais ben triste, je pleurais, pis je pleurais.

Le sentiment de solitude est ressenti par la majorité des répondants, mais de façon plus aiguë chez ceux dont l'autre parent est absent ou peu présent. II se manifeste aussi parce que le parent malade cesse les interactions positives avec l'enfant, ce dernier cessant en retour de faire des demandes au parent malade. Le deuil et l'abandon par le parent malade, la parenté ou le système de services nous ont étonnés par leur ampleur et par leurs multiples manifestations. D'abord, ces enfants ont vécu le deuil de leur parent malade, deuil d'autant plus difficile que le parent est vivant. C'est souvent par désespoir que la majorité ont fait le deuil de ce parent qui ne répond plus à leurs besoins ou attentes. De plus, la maladie semble détruire l'image du parent. Les enfants voient leurs parents dégradés, parfois violents.

Le sentiment de solitude émane aussi du discours des deuils vécus après la mort du parent malade. Les parents de quatre des participants sont décédés: deux se sont suicidés et un a été assassiné par l'autre parent, découragé à la suite de l'échec du traitement psychiatrique.

Des idées suicidaires étaient de plus présentes chez cinq autres parents malades. Dans le cas d'André et de Nathalie, il s'agit d'une double source de détresse, puisque leur père s'est donné la mort après de nombreuses menaces suicidaires et que, quelques années plus tard, leur mère a manifesté à son tour des idées suicidaires dont les enfants ont été témoins. Cette thématique du 
deuil engendre un sentiment intense de solitude et constitue un important phénomène de détresse chez l'enfant.

La solitude prend un autre sens lorsqu'elle fait suite à l'abandon ou au suicide du parent. Elle devient alors très lourde à porter et donne lieu à des sentiments ambivalents de colère et de tristesse.

Nathalie: J'étais fâchée contre mon père. Parce qu'il avait tiré sur ma mère. J'ai eu de la misère à lui pardonner. Ça m'a pris un an... On est allés au cimetière, ma mère a dit : "Y était malade, faut que tu y pardonnes, que tu vives en paix avec ça. " J'me suis mise à genoux... j'ai dit : "Papa, j'te pardonne, mais je trouve ça toujours assez dégueulasse. Si t'avais assez d'la vie, pis que maman tsé, tu y as touché, c'est... t'aurais pu t'enlever la vie tout seul sans toucher à ma mère, nous la laisser, j'te pardonne pareil. »

L'enfant ressent aussi une responsabilité, voire de la culpabilité, lorsqu'il doit penser et agir afin de "se sauver » et de sauver la fratrie. Ces situations sont plus fréquentes lorsque le parent n'est pas traité pour ses difficultés, habituellement par refus ou par négation de la maladie. Le départ des enfants du milieu familial provoque alors chez l'enfant et chez le parent un sentiment de solitude, mais permet au parent de prendre conscience de ses difficultés et d'aller chercher de l'aide. Ce moment est tout de même vécu par l'enfant comme étant très anxiogène.

Nathalie: Je ne voulais pas l'abandonner, je ne voulais pas qu'elle se sente seule... Mais c'était la meilleure solution dans le fond parce qu'elle est entrée à l'hôpital après.

La solitude est vivement ressentie lorsqu'il y a absence de soutien soit du réseau d'aide du parent, soit du milieu psychiatrique. Tous les enfants majeurs interrogés ont été ignorés par les équipes traitantes, le médecin ou l'hôpital psychiatrique. De plus, aucun de ces enfants n'a reçu de renseignements sur la maladie ou sur la façon de composer avec le parent lorsqu'il était « décompensé ».

Lucie : Moi, je savais qu'elle était malade, mais je savais pas ce qu'elle avait. On n'a eu aucune information. Aucune. Non, on n'a rien eu de ça. On n'a jamais été rencontrés par le médecin ou par quelqu'un de l'hôpital. 
Le sentiment de découragement précède habituellement celui du désespoir. Ces sentiments sont ressentis chez certaines personnes interrogées. L'enfant constate que les choses ne fonctionnent plus normalement. II est démoralisé par ce constat et, ne voyant pas de solution, il se sent alors impuissant. Parfois, malgré ses efforts pour libérer le parent de certaines responsabilités, il constate l'incapacité de celui-ci "à reprendre le dessus » et note même une détérioration des fonctions psychologiques de celui-ci.

Patrick : Pour moi euh, ça été pénible. Parce que là, euh, $y$ a rien qui ne fonctionnait plus normalement là. Aucune vie normale. Là je me sentais devenir anormal...

Dans d'autres cas, heureusement plus rares, les crises prennent des tournures catastrophiques, le parent malade ou l'entourage transmettant à l'enfant le profond découragement qu'ils éprouvent face à l'avenir.

Patrick: Le père s'est tanné. Y a été chercher un gros "beam » dans le hangar, il l'a assommée. Moi j'ai été me cacher en dessous du lit parce que... j'avais peur. Là j'ai entendu un gros cri, là j'ai vu que c'était ma mère qui criait. Mais j'osais pas aller voir dans la chambre qu'estce qui se passait. Là, mon père lui a ouvert les veines, $y$ a ouvert la gorge. Là, elle saignait abondamment, c'est sûr. Est morte au bout de son sang... Quand j'ai été au salon mortuaire le cou était couvert, les poignets étaient couverts. Là, je réalisais qu'y avait de quoi.

Le sentiment de désespoir apparaît lorsque l'enfant est retiré de son milieu naturel à cause des difficultés du parent. L'enfant éprouve une impression d'abandon de la part du parent malade, sentiment qu'il finit par surmonter lorsqu'il comprend la gravité de la maladie. Cependant, l'abandon par l'autre parent "sain » lorsqu'il quitte le domicile familial et l'abandon par la parenté ou par le système des services paraissent plus difficiles à accepter. C'est l'une des situations les plus cruelles vécues par l'enfant, car elle lui enlève tout espoir de mener une vie normale et le plonge à nouveau dans sa solitude, surtout lorsqu'il vit des difficultés dans le milieu substitut et qu'il se sent livré à son sort par les services.

Patrick : On a été placés ensemble... sauf que la madame était plus malade que ma mère. Elle nous battait... pour tout pour rien... Le travailleur social... il 
nous croyait parce qu'il voyait nos marques, mais il pouvait pas faire grand-chose. Parce qu'y avait pas de preuves contre elle. Mon frère, lui, a fugué, la police l'a retrouvé... Le travailleur social l'a pris, pis il l'a amené ailleurs. Depuis ce temps-là je vis plus avec mon frère. Je suis resté encore dans ce foyer-là pendant 7 ans. Dans l'enfer pendant 7 ans... J'étais excessivement nerveux.

Le désespoir vécu par les enfants qui vivent des situations catastrophiques peut se comprendre comme étant la perte d'espérance de voir leur situation s'améliorer. Le fait d'être abandonnés par les proches et par le système des services accentue chez l'enfant l'abattement moral vécu dans des périodes de découragement.

Ces enfants vont souvent éviter des activités ou des situations de la vie quotidienne impliquant le parent malade afin de se soustraire à des interactions avec lui ou avec son environnement. Certains adoptent un comportement irréprochable, d'autres se réfugient dans certaines activités ou même dans la maladie afin d'influencer leur relation avec leurs parents selon les attentes de ces derniers. En fait, ils développent un comportement d'évitement envers le parent attribuable, par certains d'entre eux, à une très grande docilité ou à une adaptation aux capacités du parent.

Cécile : J'essayais d'être fine, d'être sage. Vous savez quand t'es jeune, tu dis je vais être fine, pis je vais être sage; donc je serai pas battue. Mais ma mère partait dans ses états terribles où elle nous battait quand même.

Béatrice : J'étais un enfant très malade quand j'étais jeune. Je me disais que c'était un refuge ultime, la maladie.

Nicolas : Pis tu sais pas comment réagir. Pis t'oses pas emmener du monde chez vous, t'as de la misère à comprendre ça toi-même là... Tu te dis que tes amis y comprendront pas.

Les efforts de normalisation mènent parfois, en bout de ligne, à la cessation complète de demande aux parents ou à la cessation de l'expression de ses sentiments.

Béatrice : Une suradaptation... je pense que la condition sine qua non, c'est de nier totalement ses propres 
besoins comme enfant, ses propres désirs aussi, pis des émotions aussi qui sont légitimes, comme la colère, de la rage, du désespoir, de la tristesse. Alors, j'ai eu longtemps une incapacité de manifester une haute gamme d'émotions. Alors, ça fait une distorsion aussi dans sa vie affective, puis ça a distorsionné la mienne.

\section{LA MALADIE MENTALE ET LA NÉGLIGENCE PARENTALE}

Les différents phénomènes qui ressortent du corpus de données doivent être appréciés en relation avec d'autres facteurs. Nous croyons nécessaire de faire le parallèle entre les concepts de la maladie mentale et ceux de la négligence parentale afin de mieux cibler l'impact de la maladie mentale sur le fonctionnement social de l'adulte, particulièrement au regard de l'accomplissement du rôle parental. Nous retrouvons une certaine similitude entre les phénomènes de négligence parentale et de santé mentale quant aux manifestations dans le rôle parental. La littérature sur la négligence parentale nous apparaît donc pertinente pour l'analyse des phénomènes vécus selon les perceptions des difficultés du parent à accomplir son rôle parental qu'ont les enfants ayant subi cette négligence.

C'est l'aspect du psychodéveloppement qui caractérise le mieux l'état de santé mentale. Ainsi, la santé mentale d'une personne s'apprécie à sa capacité d'utiliser ses émotions de façon appropriée dans les actions qu'elle pose (affectif), d'établir des raisonnements qui lui permettent d'adapter ses gestes aux circonstances (cognitif) et de composer de façon significative avec son environnement (relationnel). (Gouvernement du Québec, MSSS, Politique de santé mentale, 1988, p. 21).

Lorsque la santé est perturbée par la maladie, le fonctionnement de la personne dans les sphères affective, cognitive et relationnelle est affecté à des degrés divers selon le type de maladie, sa durée et le degré d'atteinte. Les effets peuvent être perçus dans un ou plusieurs aspects de la vie de la personne, selon les différents rôles sociaux. Ce qui nous intéresse ici est l'impact de la maladie sur le rôle parental.

La négligence parentale se définit, selon Palacio-Quintin et Éthier (1993), comme une forme de mauvais traitement caractérisée par 
un manque de soins sur le plan de la santé, de l'hygiène corporelle, de l'alimentation, de la surveillance, de l'éducation ou des besoins affectifs, qui met en péril la croissance normale de l'enfant selon son âge et son niveau de développement. Plusieurs auteurs parlent d'échec chronique du parent à répondre aux divers besoins de l'enfant.

Les chercheurs s'entendent pour proposer trois catégories de négligence : la négligence physique, perçue comme la privation des besoins de base; la négligence développementale, renvoyant à la privation d'expériences nécessaires à la croissance et au développement, incluant la supervision et les soins pour promouvoir l'éducation, la santé (physique et mentale); et la négligence émotive, définie comme l'incapacité parentale à répondre aux besoins d'attention, de sécurité, d'estime de soi et à combler les besoins émotionnels de l'enfant (Hegar et Yungman, 1989). Contrairement aux mauvais traitements, la négligence se caractérise par «l'absence de comportements bénéfiques à l'enfant plutôt que la présence de conduites parentales néfastes, bien que, lorsque l'enfant grandit et acquiert plus d'autonomie, il est probable que les comportements du parent s'accompagnent de violence envers l'enfant » (Éthier et collab., 1994).

\footnotetext{
Certaines des conduites négligentes seraient le résultat d'un processus mental caractérisé par l'exclusion de l'information à différents stades du développement de l'enfant (Crittenden, 1993; Beck et Clark, 1988; Bowlby, 1980).
}

Sur le plan affectif, on parle d'incapacité parentale à satisfaire les besoins émotionnels de l'enfant. Sur le plan cognitif, il s'agit d'une incapacité à répondre aux besoins d'attention, de sécurité de l'enfant (Hegar et Yungman, 1989, p. 212) ainsi qu'une difficulté à trouver des solutions efficaces, des réponses ou réactions adéquates aux circonstances; le parent est dépassé par les événements (Crittenden, 1993). Sur le plan relationnel, on note une indifférence ou une grande anxiété dans la relation avec l'enfant, surtout lorsqu'il s'agit d'un très jeune enfant, et parfois le parent n'interagit pas avec lui (Browne et Sagi, 1988; Crittenden, 1993).

Nous pouvons remarquer que tant sur le plan des effets de la santé mentale que sur le plan de la négligence parentale, les diffi- 
cultés se manifestent dans les mêmes sphères, soit affective, cognitive et relationnelle. De plus, les facteurs de risque sont presque identiques pour les deux phénomènes, soit : la pauvreté économique, un environnement social chaotique, la monoparentalité, le jeune âge du parent, l'isolement social, l'aspect cognitif peu développé et la présence d'antécédents familiaux. Certains facteurs de risque reconnus en négligence parentale apparaissent comme des déterminants par rapport au développement de difficultés dans la famille ou chez l'enfant. Ces facteurs de risque sont les difficultés dans les conditions économiques et sociales, la pauvreté économique, l'environnement social chaotique, le fait que la mère soit seule pour éduquer ses enfants, le jeune âge du parent, l'isolement social, le développement cognitif pauvre et la présence d'antécédents familiaux (Brown et Sagi, 1988; Éthier et collab., 1994; Altemeir et collab., 1982; Chamberland, 1986). Aussi, les événements de stress psychosociaux auxquels l'enfant doit faire face relativement aux manifestations de la maladie et l'impact sur le fonctionnement du rôle parental sont étroitement liés à ces facteurs.

La notion de risque est une notion probabiliste qui renvoie ici aux risques plus élevés qu'a l'enfant, lorsqu'il est exposé à certaines conditions, de vivre certaines difficultés. Même si l'on précise ces facteurs de risque et ces conditions, l'appréciation exacte des chances de développer tel ou tel phénomène est en soi une aventure périlleuse. Un grand nombre d'enfants, malgré l'exposition à des facteurs de risque, se développent en grande partie normalement, même si l'on retrouve dans cette population un taux plus élevé de troubles de développement (Seifer, 1996, p. 9). D'autres ne vivront pas nécessairement des situations de négligence ou de mauvais traitements. Cette notion de risque renvoie aux concepts de facteurs de risque, de facteurs de protection, de vulnérabilité et de résilience, lesquels se juxtaposent. II est difficile d'établir une distinction précise ou une coupure entre l'un et l'autre de ces concepts. De plus, est-ce que le contraire d'une variable faisant partie des facteurs de risque constituerait un facteur de protection? Par ailleurs, la présence d'un ensemble de facteurs a une plus grande valeur prédictive; le risque est alors plus grand de retrouver tel ou tel phénomène. L'équation mathématique serait tentante pour certains. Ainsi, la plupart des recherches vont indiquer que ce sont les effets combinés de multiples 
facteurs de risque qui expliqueraient l'apparition de difficultés plutôt que des facteurs isolés. L'approche écosystémique inspire de plus en plus de chercheurs intéressés aux problèmes développementaux et psychosociaux chez ces enfants.

Les travaux de Garmezy (1984) et de Masten (1989) ont fait avancer ces connaissances appliquées au domaine de la maladie mentale et ils ont soulevé la notion de résilience chez les enfants qui, malgré l'exposition aux facteurs de risque reconnus, se développent "normalement ». Cette notion de résilience référerait à un processus interne chez l'enfant par rapport aux facteurs externes impliquant ces facteurs de risque comme élément explicatif. Ces enfants résilients ou non vulnérables aux facteurs de risque externes présents dans leur environnement se développeraient normalement parce que les facteurs de protection leur sont internes, c'est-à-dire propres à leur personne et à leurs qualités personnelles. La compréhension de ces notions et le développement des connaissances quant aux facteurs de risque, aux facteurs de protection et à la résilience sont incontournables si l'on veut éviter l'accroissement des problèmes psychosociaux de ces enfants et de leurs parents (Seifer, 1996, p. 16).

Dans le cadre de cet exercice, nous avons regroupé les perceptions des manifestions de la maladie du parent sous les thématiques : rôle parental / manifestations sur les plans affectif, cognitif, relationnel et conditions sociales afin de reprendre les catégories de manifestations que l'on a vues plus haut.

\section{RÔLE PARENTAL}

Dans le discours de nos répondants, nous avons pu reconnaître l'incapacité du parent malade à répondre aux besoins émotionnels de son enfant. Cette manifestation correspond à la négligence émotive définie comme l'incapacité parentale à satisfaire les besoins d'attention, de sécurité, d'estime de soi et à combler les besoins émotionnels de l'enfant (Hegar et Yungman, 1989). Pour certains enfants, on peut parler d'une absence totale de rapports affectifs. Pour les autres, la réponse à ces besoins affectifs est déficiente chez la majorité des personnes interrogées. 
Béatrice : Je vous dirais que pour ma part la relation affective, du moins avec ma mère, n'était pas très présente.

Cécile : Ma mère n'en donnait pas, d'affection... C'est pour ça que quand on arrivait chez ma grand-mère, c'était un cadeau d'avoir un bec avant de se coucher, d'être bordé. Ça on l'avait pas et moi je le recherchais beaucoup en tout cas.

Les conduites sur le plan cognitif démontrent dans certaines situations révélées aussi par le discours une incapacité à répondre aux besoins d'attention et de sécurité de l'enfant. Les personnes interrogées témoignent de réponses ou de réactions du parent inadaptées aux circonstances et laissent entendre que le parent a eu des difficultés à trouver des solutions efficaces et qu'il semblait dépassé par les événements.

Toutes les personnes interrogées ont vécu des situations où le parent, à cause de son état mental, a éprouvé des difficultés de façon ponctuelle, prolongée ou chronique à avoir un raisonnement lui permettant d'adapter de façon appropriée ses gestes aux circonstances. Les décompensations avaient un effet sur ces capacités.

Nicolas : ... tu te fais réveiller en pleine nuit pour voir si on est vivant... Tu te rends compte que ta mère est pas normale.

Béatrice : J'avais même pas eu le temps de manifester quoi que ce soit. Elle m'a dit: T'es en pénitence parce que, ton aura, il y manque des ondes... Bref, à partir d'un élément qui n'était pas encore arrivé dans la réalité, même pas une faute commise. Là, ça a nécessité une punition. Bon, d'aller dans sa chambre, c'est quand même pas des blessures physiques, mais c'est au plan de la confusion entre le réel et le délire des parents.

Les comportements du parent en décompensation sont des stresseurs importants et provoquent des inquiétudes chez l'enfant qui est, à la phase aiguë de la maladie, témoin de crises. II conçoit des stratégies d'adaptation, mais parfois le climat familial dégénère à tel point que tous les membres de la famille deviennent vulnérables. 
Cécile : J'ai été témoin aussi qu'elle prenait mon père pour une autre personne. Vous savez, s'il arrivait des choses, qu'elle entendait à la radio, comme un avion qui a tombé, qu'une personne l'a fait sauter, ma mère a pouvait prendre mon père pour la personne qui a fait sauter l'avion. Là, on comprenait pas. On pouvait pas comprendre ça. Donc, elle devenait encore très agressive. Parce que mon père aussi a été battu. On dirait qu'elle avait comme un pouvoir sur tous nous autres.

On a aussi pu voir que plusieurs répondants ne comprenaient ni les événements ni la maladie. Certains enfants vont dire que c'est beaucoup plus tard, à l'âge adulte, qu'ils ont appris que telle ou telle chose était anormale ou même nuisible et attribuable à la maladie de leur parent.

\begin{abstract}
Béatrice : ... tout l'impact aussi de disputer toujours autour de thèmes portant sur la fin du monde, sur les esprits. J'allais à l'école et puis quand je parlais avec mes petites amies, c'était pas, on joue-tu aux élastiques; c'est, savais-tu que la fin du monde s'en vient (délire du parent). Là, le monde me regardait comme un extraterrestre. Ça, c'était mon vécu familial de tous les jours, enrobé aussi dans un discours qui pouvait le légitimer, dans le sens que mes parents étaient très religieux.
\end{abstract}

La perception de la réalité chez l'enfant peut être perturbée par la présence d'un délire paranoïde du parent qui sent des complots dirigés contre lui et, parfois, ce délire inclut l'enfant.

Béatrice : Lorsqu'elle a décompensé, le délire était plus apparent, un délire à saveur paranoïde où le FBI était à ses trousses, il fallait parler tout bas, parce qu'on pouvait être entendu, puis il y avait un complot contre nous autres.

Les conduites sur le plan relationnel semblent montrer que la majorité des personnes interrogées ont vécu des situations où le parent, à cause de son état mental, a éprouvé des difficultés à composer avec l'environnement d'une manière appropriée, de façon ponctuelle, prolongée ou chronique.

Lucie : Elle nous a renfermés dans des garde-robes. Pourquoi? Je sais pas. Elle nous faisait peur aussi, elle nous cachait en dessous des lits. Parce que les sœurs 
(religieuses) passaient. Ça sonnait à porte... on se cachait en dessous des lits.

En ce qui concerne le rôle du parent, ces manifestations sur le plan relationnel se traduisent par une indifférence ou par une grande anxiété dans sa relation avec l'enfant, surtout lorsqu'il s'agit d'un très jeune enfant, ou encore par une absence d'interaction entre le parent et son enfant.

Nathalie : Un rien la bousculait. Fallait pas que tu fasses quelque chose de travers, elle était beaucoup fatiguée, elle paniquait à rien. Dans ce temps-là elle s'occupait pas beaucoup de mon frère. Elle était vraiment fatiguée, agressive, vraiment triste, ça n'allait pas bien.

Lucie: Mais ma mère ne voulait plus rien savoir de nous autres, de personne, ni des enfants, ni du mari.

Le cumul de ces manifestations, combiné au manque de soutien familial, semble entraîner une escalade de problèmes menant à la violence familiale. L'enfant le plus âgé va souvent se sacrifier pour protéger les plus jeunes.

Lucie : J'étais la plus vieille, j'essayais de prendre ça sur mon dos, j'essayais de l'entourer, de l'aider pour pas qu'elle fasse mal à mes sœurs. J'en ai reçu des claques, des coups pour les autres. Je l'entourais. Quand je voyais qu'elle "feelait » pas bien, je m'approchais d'elle, j'allais la voir pour pas qu'a vienne voir mes deux sœurs.

Enfin, l'inversion du rôle parental constitue une autre difficulté relationnelle. On remarque, particulièrement chez les enfants de sexe féminin les plus âgées de la fratrie et dans les familles où le père est peu présent, le développement d'une autonomie excessive qui ne va pas sans conflit avec le parent malade qui se sent parfois menacé. Parmi les personnes de sexe féminin interrogées, cinq sur six ont vécu ce phénomène.

Nathalie: Je me sentais avoir plus de responsabilités dans la maison, prendre plus : faire mes devoirs, les devoirs de mon frère... et je protégeais mon frère tout le temps. C'est moi qui donnais les ordres à mon frère, ma mère ça la fâchait, a sentait qu'a prenait moins de place, ça faisait beaucoup de chicane. Je savais que ma mère n'en pouvait plus... Je le sentais. Je sais que ma mère aimait pas trop ça, mais j'avais pas le choix dans le fond. 
Le discours révèle que ces symptômes ont pu être atténués ou exacerbés par des facteurs de risque de nature sociale : pauvreté économique, environnement social chaotique, monoparentalité, jeune âge du parent, isolement social, aspect cognitif peu développé, présence d'antécédents familiaux; de nature environnementale: parenté, services psychiatriques, services dans la communauté; et par des facteurs personnels liés à l'enfant. II semble, selon les témoignages de ces enfants, que les facteurs de risque pourraient prendre une plus grande importance lorsqu'il y a présence de maladie mentale d'un parent. L'analyse des informations recueillies lors des entrevues nous amène à constater que, parmi les onze personnes interrogées, huit ont vécu dans une famille où quatre à sept des facteurs de risque étaient présents. L'isolement social de la famille, particulièrement de l'enfant, semble jouer un rôle des plus importants dans la manifestation de difficultés associées à l'accomplissement du rôle parental. Ce même facteur contribue aussi à la détresse psychologique de l'enfant, qui n'a parfois personne à qui se confier ou qui n'a tout simplement pas « l'autorisation du parent " pour le faire.

L'isolement social peut être attribuable à l'attitude du parent malade, mais aussi à l'absence de soutien de l'environnement, y compris celui des services psychiatriques. Dans le prochain extrait, on retrouve au moins trois des sept facteurs de risque : la pauvreté économique; un environnement social chaotique et l'isolement social. Nous savons par ailleurs que les deux parents concernés dans cet extrait avaient moins de vingt ans à la naissance de leur premier enfant et n'avaient fait que des études de niveau primaire.

Cécile: C'étaient des conditions de vie pauvres, c'étaient des conditions de vie difficiles, ma mère malade et mon père qui buvait beaucoup. Mais le plus dur c'est parce que ma mère était une personne qui nous laissait pas sortir, pis j'en voulais beaucoup à ma mère pour ça.

Dans le prochain extrait, cinq des sept facteurs de risque sont présents dans la famille, soit : la pauvreté économique, un environnement social chaotique, la monoparentalité, l'aspect cognitif peu développé du parent et l'isolement social vécu par l'enfant en raison de l'absence de soutien du parent ou par négligence com- 
munautaire des services psychiatriques. À noter par ailleurs que les deux parents dont il est question dans le prochain extrait avaient moins de vingt ans à la naissance de leur premier enfant, qu'ils avaient terminé leur primaire seulement et vivaient dans des conditions économiques difficiles.

Denise : J'ai quand même été élevée toute seule. C'était débrouille-toi. Mon père était parti parce qu'ils se chicanaient tout le temps. Ma mère était pas capable de donner des explications de quoi que ce soit... Informe-toi (à qui?)... parce que maman nous donnait pas les renseignements nécessaires, tu sais. Aussi, même si on savait qu'elle était malade parce qu'elle était allée à SaintJean-de-Dieu... jamais personne nous a expliqué ce qu'elle avait, ni comment agir avec elle ou par rapport aux problèmes que ça nous donnait.

\section{Discussion}

Un des avantages de la méthode de recherche qualitative est de favoriser l'émergence des connaissances à partir du corpus qualitatif des données. Rappelons qu'un des désavantages de cette méthode est qu'elle permet difficilement la vérification des résultats et la généralisation de ceux-ci. Le petit nombre de personnes interrogées au cours de cet exercice et le fait que l'échantillon soit composé de volontaires dont certains ont souffert eux-mêmes d'une maladie mentale nous amènent à insister davantage sur ces limites de généralisation.

L'examen de ces témoignages permet cependant d'attirer l'attention sur certains indices de détresse psychologique et sur le risque, pour les enfants qui vivent avec un parent ayant une maladie mentale, de connaître des situations de négligence parentale. La première partie de l'analyse, qui porte sur les situations particulières vécues par les enfants lors de manifestations aiguës de la maladie mentale de leurs parents, en lien avec certaines dimensions de la détresse psychologique, fait ressortir que toutes les personnes interrogées semblent, à des degrés divers, avoir connu des situations de détresse psychologique. Ces indices de détresse se manifestent de plusieurs façons : - la peur autour des manifestations de la maladie mentale du parent, peur de la décompensation, de la rechute, de la détérioration de la 
situation du parent faisant parfois craindre le pire; - la tristesse devant l'impuissance du parent à s'en sortir, devant la propre incapacité de l'enfant à prendre la relève, devant le suicide ou les gestes suicidaires du parent, lesquels provoquent de grandes peines; - la solitude due à la cessation ou à l'absence d'interaction avec le parent, qui correspond à une forme de deuil du parent ne répondant plus à ses besoins, solitude due également à l'absence de soutien de l'environnement, y compris le réseau d'aide psychiatrique; - le découragement et le désespoir devant l'ampleur des problèmes et devant la dégradation de la situation du parent et de la famille ainsi que l'abandon ressenti; - des comportements d'évitement envers le parent malade et l'entourage.

La deuxième partie, qui porte sur les difficultés pour les parents souffrant de maladie mentale d'accomplir leur rôle parental, en lien avec les dimensions de la négligence parentale et de la maltraitance, fait état d'indices de négligence dans le rôle parental et révèle que les parents semblaient être affectés dans leur capacité parentale à des niveaux de gravité variables. Les situations vécues par la majorité des répondants semblent avoir compromis leur bien-être, leur sécurité et leur développement à divers degrés et pendant des périodes de temps variables.

Les indices de la détresse psychologique que vit l'enfant d'une personne atteinte de maladie mentale et les manifestations associées à l'exercice du rôle parental semblent fortement interreliés. Cette observation que certains jugeront évidente donne une couleur aux affirmations selon lesquelles les enfants de parents souffrant de difficultés psychopathologiques ${ }^{3}$ ont dix fois plus de chances que d'autres d'éprouver les mêmes difficultés psychopathologiques que leurs parents ou d'autres troubles psychopathologiques (Beardslee et collab., 1983). Ces enfants seraient exposés à des risques beaucoup plus grands que la population en général de vivre des problèmes au cours de leur enfance ainsi qu'à l'âge adulte. De plus, ils risqueraient davantage de subir de la négligence parentale, des mauvais traitements, d'éprouver des problèmes de développement, d'être aux prises avec des problèmes

3 La notion de psychopathologie doit être comprise comme étant un terme qui signifie une maladie mentale au sens des critères diagnostiques du DSM-IV ou une dépendance à une substance toxique ou psychoactive. 
psychosociaux et environnementaux (Seifer, 1996; Lacharité, 1992) ou de subir davantage des transformations familiales. Par ailleurs, certains seraient protégés de l'apparition de ces problèmes en raison de la présence de facteurs de protection permettant de limiter l'impact des effets négatifs sur leur bien-être ou sur leur développement. D'autres vivraient plus de problèmes, ce qui aurait un impact dans l'apparition d'autres troubles éventuels. L'exposition à ces facteurs de risque, la présence de plusieurs facteurs à la fois, l'accumulation de ces facteurs et l'absence de facteurs de protection pouvant combler certains manques ne seraient pas les seuls éléments à considérer dans ces situations. L'enfant exposé à toutes ces difficultés ne vivrait pas nécessairement de problèmes dans l'enfance ou à l'âge adulte. Malgré cette exposition aux facteurs de risque, certains enfants n'éprouveraient pas de difficultés. Ces notions de facteurs de risque, de facteurs de protection et de résilience peuvent aider à mieux comprendre certains phénomènes. Cependant, ces prétendues évidences devraient attirer l'attention autant des chercheurs que des cliniciens et des établissements dispensateurs de services sur la nécessité d'harmoniser leurs efforts sur le plan de la santé publique.

L'analyse des situations vécues par ces enfants nous amène donc à porter une attention particulière à ces facteurs de risque qui, rappelons-le, constituent des données probabilistes. Sans vouloir faire une adéquation mathématique, il nous semble cependant que certaines dimensions devraient être considérées lors de l'évaluation diagnostique lorsque l'on cherche à déterminer le risque de détresse de l'enfant qui est dans ces situations ou lorsque l'on tente de déterminer les manifestations de négligence parentale dans ces familles. Le cumul des conditions adverses, leur degré de gravité, la durée et les répétitions semblent susceptibles d'augmenter le risque, pour l'enfant de la personne atteinte de maladie mentale, de vivre de la détresse psychologique ainsi que de subir des situations de négligence parentale. Plus il y a présence de conditions adverses, plus les difficultés pourraient être élevées. La durée, la gravité et la répétition des difficultés dues à ces conditions adverses paraissent affecter négativement les chances de ces enfants d'avoir une bonne santé mentale durant l'enfance et pourraient augmenter leurs risques de souffrir d'une maladie mentale. 
Les conditions adverses seraient des facteurs liés à la maladie du parent, aux conditions de réussite de la réadaptation psychosociale, particulièrement la dimension du rôle parental, et aux facteurs de risque en présence.

Il semble, selon ce qui ressort de ces témoignages, que les facteurs liés aux particularités de la maladie mentale du parent pourraient constituer une dimension très vaste de facteur d'influence, mais retenons, par ordre d'importance, ce dont l'enfant peut être témoin ou victime : le type de maladie, le type de décompensation, les manifestations de la maladie, les crises. L'enfant témoin de l'état psychologique du parent vivra des situations différentes selon le type de psychopathologie. Aussi, la durée de la décompensation ou des difficultés de fonctionnement du parent, la répétition des rechutes, les capacités du parent dans l'ensemble de ses rôles sociaux durant les périodes de rémission semblent également des facteurs d'influence à considérer. Tous les symptômes liés à la maladie exerceraient des effets sur le fonctionnement psychosocial, entre autres sur l'accomplissement du rôle parental, mais aussi sur l'ensemble des interactions sociales, lesquelles il faudrait quantifier (durée, fréquence) et qualifier (gravité). Ces situations auraient aussi un impact sur les sentiments vécus par l'enfant en rapport avec la maladie du parent.

Les conditions de réadaptation en psychiatrie, dont l'obtention d'une médication appropriée, l'accessibilité aux services dans les situations de crise, la possibilité d'une intervention familiale de type psychoéducatif, la possibilité et l'acceptation d'un suivi à long terme (Tessier et collab. 1992, p. 210), sont essentielles à ces familles. L'impact des difficultés est lié à la possibilité de recevoir un traitement ou de profiter d'un service disponible dans la communauté, mais il dépend aussi du désir du parent de recevoir traitement ou services. Plusieurs répondants témoignaient en effet du déni des difficultés et du refus du parent de recevoir des soins, à cause de croyances ou de préjugés. Ces conditions devraient, selon nous, tenir compte de certaines attitudes du parent, soit : la reconnaissance nécessaire de la maladie, par lui-même et par ses proches, l'adhésion et l'observance du traitement proposé, y compris la médication. Tessier abonde dans le même sens : 
La réadaptation, dans le sens étroit du terme, implique que la personne veut bien se placer dans une situation de développement d'habiletés, c'est-à-dire dans un processus d'apprentissage. Or, tel n'est pas toujours le cas (Tessier et collab., 1992, p. 13).

Aussi, les modèles d'intervention traditionnels tiennent peu compte du rôle parental des personnes atteintes de maladie mentale. Dans la littérature, lorsqu'on parle de famille, on fait référence à la fratrie et aux parents. Très peu d'écrits ont jusqu'à maintenant traité de la dimension du rôle parental. Or, les dimensions du rôle parental des personnes atteintes de maladie mentale, la préoccupation face à ce que vivent leurs enfants dans les phases aiguës de la maladie de leurs parents et l'implication de l'enfant dans l'ensemble du processus d'aide devraient préoccuper davantage autant les chercheurs que les intervenants. Même s'ils sont restreints en nombre, les témoignages des personnes interrogées dans le cadre de cette étude démontrent que ces dimensions semblent avoir fait plus que défaut pour elles.

Les "facteurs de risque " présents dans les familles où un parent souffre de maladie mentale semblent accroître les risques chez l'enfant de vivre des situations de détresse psychologique et de subir des situations de négligence parentale.

Lorsque l'on tente de dégager certaines pistes d'intervention qui pourraient aider ces familles, il nous faut admettre que certains des facteurs de risque, comme la pauvreté économique, la monoparentalité, le jeune âge du parent, la présence d'antécédents familiaux, sont " peu traitables ", alors que l'intervention qui favorise un meilleur soutien social et, parfois, l'amélioration des conditions matérielles d'existence peut donner des résultats tangibles. Nous devons consentir à des efforts d'intervention visant à atténuer l'isolement social dans l'environnement immédiat, à mettre en place un réseau de soutien social incluant celui que peut apporter l'équipe traitante et à soutenir le parent et l'enfant dans leurs difficultés propres. Des efforts spécifiques axés sur la transmission de connaissances (aspect cognitif) à la personne aux prises avec ces difficultés, à ses enfants et à ses proches pourraient avoir un effet positif. II conviendrait, par exemple, de leur donner de l'information sur la maladie, sur ses effets, sur le fonctionnement en général, y compris le rôle parental, sur les médicaments 
et leurs effets, sur les traitements et les services d'aide disponibles dans la communauté, pouvant prévoir avec eux des mécanismes de soutien en cas de crise.

Par ailleurs, nous croyons, en nous référant à la littérature sur la négligence parentale, que ces familles ou ces parents se distinguent en plusieurs points. Ainsi, la "capacité parentale » est parfois atténuée à cause de la maladie ou réduite ponctuellement de façon plus ou moins marquée lors d'une décompensation. Plusieurs de ces parents retrouvent leur pleine capacité de fonctionnement social, y compris le rôle parental, dans les périodes de rémission contrairement à la "compétence parentale ", qui a un caractère plus permanent. Aussi, lorsqu'un enfant manque de soins ou est négligé par le parent lors d'une phase aiguë de la maladie, nous devons parler de négligence transitoire. De plus, la manifestation de certains comportements ou l'absence de comportements bénéfiques aux besoins de l'enfant ne signifient pas que l'enfant est négligé automatiquement; il peut l'être par un parent sans l'être par l'autre conjoint.

L'enfant d'un parent qui souffre de maladie mentale n'est pas nécessairement en "situation de compromission » au sens de la Loi sur la protection de la jeunesse (Gouvernement du Québec, 1996), car plusieurs facteurs influencent l'analyse de sa situation, comme ceux liés aux ressources personnelles de l'enfant, aux ressources sociales du milieu et aux conditions sociales de la famille.

L'approche écologique facilite la compréhension de situations humaines en tenant compte de plusieurs facteurs qui ont une influence entre eux. Notre étude sur les phénomènes subis dans l'enfance par des personnes qui ont vécu avec un parent souffrant de maladie mentale et sur les difficultés quant à l'accomplissement du rôle parental de leur parent tente de démontrer certains de ces facteurs d'influence. La maladie du parent n'est pas seule en cause. Certaines conditions peuvent influencer l'impact sur le rôle parental, allant de caractéristiques individuelles comme la non-reconnaissance ou le déni de la maladie au manque de connaissances, à certaines conditions sociales, particulièrement: l'isolement social, la pauvreté économique, l'environnement social chaotique, la monoparentalité, la présence d'antécédents familiaux, mais aussi le système et les politiques sociales 
comme l'absence de programmes d'intervention spécialisés et de soutien pour ces familles vulnérables. Les indices de détresse psychologique vécue par ces enfants et les situations de négligence - bien qu'elles soient parfois transitoires - demeurent tout de même exacerbés par un ensemble de facteurs qui ne sont pas attribuables uniquement à la maladie du parent ou à d'autres caractéristiques personnelles du parent.

L'approche écologique suggère de saisir le phénomène "à tous ses niveaux systémiques sans trahir sa complexité " (Mayer, 1997, p. 215) pour mieux cibler les actions et ainsi éviter que l'intervention ne repose que sur une approche individuelle auprès du parent, lequel porte, à ce moment, toute la responsabilité de la situation (Mayer, 1997).

\section{CONCLUSION}

Cette étude étant limitée quant au nombre de personnes interrogées et quant à leur origine (milieu clinique), elle favorise tout au plus un certain défrichage d'informations qui donnent la possibilité de formuler certaines hypothèses de recherche. Malgré ces limites, elle permet d'attirer l'attention sur les indices de détresse psychologique et de négligence parentale que ces enfants risquent de vivre dans certaines conditions. Des phénomènes qui pouvaient paraître, de prime abord, marginaux ou exceptionnels semblent plus importants que ceux prévus et ont été mis au jour, notamment: la négligence parentale, le phénomène de mauvais traitements, l'inversion du rôle parental, l'exclusion de l'enfant du processus d'aide, les indices de détresse incluant les deuils importants, voire la mortalité du parent malade.

Des pistes de solution pourraient, selon nous, atténuer le risque de négligence parentale dans certaines conditions. La première condition est de s'assurer que le parent est traité, car il augmenterait ainsi ses chances de "retrouver " ses capacités affectives, cognitives ou relationnelles. La deuxième est d'obtenir le soutien de l'autre parent ou la présence d'un substitut parental stable. La troisième est d'assurer la présence d'une équipe traitante qui tiendra compte de la situation parentale de la personne traitée et qui inclura les enfants dans le traitement. Dans ces conditions, les risques de négligence parentale peuvent s'atténuer, la personne 
retrouvant ses facultés et pouvant à nouveau exercer son rôle de parent de manière conforme et adaptée aux besoins de ses enfants. Dans le cas où le parent est atteint sur le plan des compétences parentales, la condition psychiatrique n'est pas l'élément déclencheur des difficultés parentales: elle n'a qu'exacerbé un état déjà perturbé.

L'absence de considération du rôle parental des personnes atteintes de maladie mentale, le manque d'information sur la maladie, l'absence de lien avec l'équipe ainsi que l'absence d'enseignement de moyens et d'ententes intrafamiliales en cas de décompensation sont des facteurs qui contribuent à la fragilisation de l'équilibre familial, selon nous. La conspiration du silence nous semble cependant, parmi ces phénomènes, l'un des plus déterminants, car toutes les personnes interrogées ont parlé de cette absence d'information et de soutien. La littérature sur les conditions de réussite de la réadaptation en psychiatrie souligne pourtant l'importance d'offrir du soutien et de l'information sur la maladie à tous les membres de la famille afin de maintenir l'équilibre familial. Les personnes interrogées se sont particulièrement senties seules, sans moyens et abandonnées à leur sort par les services psychiatriques. Cette attitude constitue un type de négligence appelé la négligence communautaire et est attribuable à un certain désintérêt des ressources professionnelles relativement à la situation des enfants à qui elles offrent ou devraient offrir des services (Ménard et Pinard, 1987, p. 4).

Plusieurs des personnes interrogées (les deux tiers) ont ellesmêmes souffert d'un problème de santé mentale à l'âge adulte et nous avons pu constater au cours de leurs témoignages que plusieurs facteurs déterminants quant au développement d'un problème de santé mentale ont été présents dans leur vie. L'exposition des enfants à ces facteurs a-t-elle pu compromettre leur développement et fragiliser leur propre santé mentale?

Les témoignages des personnes qui ont vécu avec un parent souffrant de maladie mentale nous ont permis de mieux cerner les facteurs de risque de négligence parentale en psychiatrie et d'identifier ce qui compromet et contribue à la chronicité des situations de négligence. Bien souvent, le caractère chronique des 
situations de négligence est attribuable à d'autres facteurs que la seule responsabilité individuelle du parent malade.

La transformation du réseau de services en santé mentale au Québec et la suppression de lits dans le milieu psychiatrique risquent d'avoir des conséquences majeures insoupçonnées sur le fardeau familial et encore plus si les personnes atteintes de maladie mentale ont des enfants mineurs. Ces nouvelles contraintes risquent, dans certains cas, de compromettre davantage la sécurité et le bien-être de leurs enfants mineurs. Selon nous, un effort spécial doit être fait par les intervenants et les décideurs des programmes de services afin de soutenir ces familles.

Marc BOILY

Travailleur social Hôpital Louis-H. Lafontaine

Étudiant au Ph. D. (service social) à l'Université Laval

Viviane LEW

Médecin et psychiatre exerçant aux hôpitaux Jean-Talon et Louis-H. Lafontaine

Chef du département de psychiatrie de l'Hôpital Jean-Talon

Professeure adjointe de clinique Département de psychiatrie de l'Université de Montréal

Pauline MORISSETTE Professeure agrégée École de service social de l'Université de Montréal Chercheure principale dans l'équipe de recherche et intervention sur les substances psychoactives - Québec (RISQ) 


\section{Références bibliographiques}

Altemeir, W., S. O'Connor, P. Victze, H. SANDleR et K. SherRod (1982). «Antecedents of Child Abuse», Journal of Pediatrics, vol. 100, p. 823-829.

ANTHONY, E.J. (1987). "Risk, vulnerability and resilience. An overview, II », dans E.J. Anthony et B.J. Cohler, The Invulnerable Child, New York, The Guilford Press.

ANTHONY, E.J. (1976). " How children cope in families with a psychotic parent », dans E.N. Rexford, L. Sander et T. Shapiro (dir.), Infant Psychiatry: A New Synthesis, New Haven, Yale University Press.

ANTHONY, E.J. (1969). " A Clinical Evaluation of Children with Psychotic Parents », American Journal of Psychiatry, vol. 126, p. 177-184.

Beardslee, W.R., J. Bemporad, M.B. Keller et G.L. KLeRman (1983). "Children of Parents with Major Affective Disorders: A Review », American Journal of Psychiatry, vol. 140, $n^{\circ} 7$, p. 825-832.

BECK, A.T. et D.A. CLARK (1988). " Anxiety and depression : An information processing perspective ", Anxiety Research, vol. 1, p. 23-36.

Bellerose, C., E. Lavallée et J. Camirand (1994). Enquête sociale et de santé 1992-1993. Faits saillants, Québec, Santé Québec, ministère de la Santé et des Services sociaux.

Blanch, A., J. Nicholson et J. PuRCELl (1994). «Parents with Severe Mental Illness and Their Children: The Need for Human Services Integration », The Journal of Mental Health Administration, vol. 21, p. 388-396.

BLANCH, A., et R. SURLES (1993). "Case Management interventions in rehabilitation », dans J. Bedell (dir.), Psychosocial Assessment and Treatment of Patients with Severe Mental Disorders, Washington, Taylor and Francis.

BoILY, M. et V. LEW (1997). « La négligence parentale en psychiatrie... la prévenir », Intervention, no 105, p. 18-29.

BoWLBY, J. (1980). Attachement et perte, vol. 2 : La séparation, angoisse et colère. Traduit de l'anglais par Didier E. Weil (1984), Paris, Presses universitaires de France. 
BRETON, J.J., L. BERGERON et J.P. VALLA (1992a). Enquête québécoise sur la santé mentale des jeunes de 6 à 14 ans, Faits saillants, Prévalence des troubles mentaux et utilisation des services, Hôpital Rivière-des-Prairies et Santé Québec.

Breton, J.J., L. Bergeron, J.P. Valla, C. Berthiaume, N. Gaudet, J. LAmBert, M. St-Georges, L. Houde, S. Lépine et C. DAVeluy (1992b). Enquête québécoise sur la santé mentale des jeunes de 6 à 14 ans, vol. 2, Prévalence des troubles mentaux et utilisation des services, Hôpital Rivière-des-Prairies et Santé Québec.

BROWNE, K. et S. SAGI (1988). "Approaches to Screening for Child Abuse and Neglect ", dans K. Browne, C. Davies et P. Stratton (dir.), Early Prediction and Prevention of Child Abuse, Rochester, John Wilez \& Sons.

Chamberland, C., C. Bouchard et J. Beaudry (1986). "Conduites abusives et négligences envers les enfants: Réalités canadienne et américaine ", Revue canadienne des sciences du comportement, vol. $18, n^{\circ} 4$.

COMMISSION DES DROITS DE LA PERSONNE ET DES DROITS DE LA JEUNESSE (1998). Rapport d'enquête : Décision recommandations, le cas des enfants maltraités de Beaumont, Québec.

CONSEIL DE LA SANTÉ ET DES SERVICES SOCIAUX DE LA RÉGION DU MONTRÉAL MÉTROPOLITAIN (1990). Plan d'organisation des services de santé mentale de la région du Montréal métropolitain, 19901995.

CRITTENDEN, P. (1993). « An Information-Processing Perspective on The Behavior of Neglectful Parents », Criminal Justice and Behavior, vol. 20, p. 27-48.

EKDAHL, M., E. RICE et T.W. SCHMID (1962). "Children of Parents Hospitalized for Mental Illness », American Journal of Public Health, vol. 52, p. 428-435.

Éthier, L., J.-P. Gagnier et C. LACHARItÉ (1994). "Prévenir la négligence parentale ", Revue québécoise de psychologie, vol. 15, $\mathrm{n}^{\circ} 3$, p. 67-86.

GARMEZY, N. (1974). "Children at Risk: The Search for Antecedents of Schizophrenia, Part 1, Conceptual Models and Research Methods ", Schizophrenia Bulletin, vol. 8, p. 14-90.

GARMEZY, N. (1984). "Children Vulnerable to Major Mental Disorders, Risk and Protective Factors ", dans L. Grinspoon (dir.). Psychiatry Updates, Washington, DC, American Psychiatric Press, p. 91-120. 
GARMEZY, N. (1984b) «Stress-resistant Children : The Search for Protective Factors ", dans J.E. Stevenson (dir.), Recent Research in Developmental Psychopathology, Journal of Child Psychology and Psychiatry, Book Supplement No. 4, p. 213-233.

GOUVERNEMENT DU QUÉBEC (1998). Plan d'action pour la transformation des services en santé mentale, Québec, ministère de la Santé et des Services sociaux.

GOUVERNEMENT DU QUÉBEC (1997). Bilan d'implantation de la politique en santé mentale, Québec, ministère de la Santé et des Services sociaux.

GOUVERNEMENT DU QUÉBEC (1997). Défis de la reconfiguration des services en santé mentale, Québec, ministère de la Santé et des Services sociaux.

GOUVERNEMENT DU QUÉBEC (1997). Orientation pour la transformation des services de santé mentale, Document en consultation, Québec, ministère de la Santé et des Services sociaux, Direction de la planification et l'évaluation.

GOUVERNEMENT DU QUÉBEC (1996). Loi sur la protection de la jeunesse, L.R.Q., chap. P-34.1, Québec, ministère de la Santé et des Services sociaux.

GouvernemenT DU QUÉBEC (1988). Politique de santé mentale, Québec, ministère de la Santé et des Services sociaux.

Grünebaum, H.U., B.J. Cohler, C. Kauffman et D. Gallant (1978). "Children of Depressed and Schizophrenic Mothers », Child Psychiatry and Human Development, vol. 8, $\mathrm{n}^{\circ}$ 4, p. 219-228.

Guberman, N., P. MAheu et C. MAillé (1993). Et si l'amour ne suffisait pas..., Montréal, Les Éditions du remue-ménage.

Hatfield, A.B. (1978). "Psychological Cost of Schizophrenia to the Family », Social Work, septembre, p. 355-359.

Hegar, R.L. et J.J. Yungman (1989). "Toward a Causal Typology of Child Neglect ", Children and Youth Services Review, vol. 11, p. 203-220.

HibBs, E.D., S.D. Hamburger, M. LenANe et J. RAPOPORT (1991). " Determinants of Expressed Emotion in Families of Disturbed and Normal Children », Journal of Child Psychology and Psychiatry and Allied Disciplines, vol. 32, $\mathrm{n}^{\circ}$ 5, p. 757-770. 
InOFF-Germain, G., E.D. NotTelmanN et M. RadKe-YARROW (1992). «Evaluation Communications Between Affectively III and Well Mothers and Their Children ", Journal of Abnormal Child Psychology, vol. 20, p. 189-212.

KAPLAN, H.I., J.B. SADOCK et R. CANCRO (1998). Synopsis of Psychiatry : Behavioral Sciences : Clinical Psychiatry ( $8^{\mathrm{e}}$ éd.), Baltimore, Williams \& Williams.

Kaufman, C., H.U. Grünebaum, B.J. Cohler et E. Gamer (1979). "Superkids : Competent Children of Psychotic Mothers », American Journal of Psychiatry, vol. 136, p. 1398-1492.

KIRSH, S. (1989). "Sommes-nous à l'écoute des enfants d'adultes atteints de troubles mentaux? ", Santé mentale au Canada, vol. 37, p. 29-30.

LAFOND, C. (1993). Avoir une mère atteinte de maladie mentale: trois histoires de vie. Mémoire présenté à la Faculté des études supérieures en vue de l'obtention du grade de Maître ès sciences (M.Sc.), Université de Montréal, Montréal.

LEW, V. et M. Bolly (1999). "Les risques psychosociaux chez les enfants de personnes atteintes de maladie mentale ", dans $\mathrm{E}$. Habimana, L. Éthier, D. Petot et M. Tousignant, Psychopathologie de l'enfant et de l'adolescent: Approche intégrative, Boucherville, Gaëtan Morin.

MAYER-RenAUD, M. (1990). Le phénomène de la négligence, Centre des services sociaux du Montréal métropolitain.

MAYER, M. (1997). Les contextes écologiques d'incidence de mauvais traitements à l'égard des enfants dans la région de Montréal. Thèse présentée à la Faculté des études supérieures en vue de l'obtention du grade de Philosophiae Doctor (Ph. D.) en sciences humaines appliquées, Université de Montréal, Montréal.

MAYER, R. et F. OUELLET (1991). Méthodologie de recherche pour les intervenants sociaux, Boucherville, Gaëtan Morin.

MAZIADE, M. (1990). "Études sur le tempérament : Contribution à l'étude des facteurs de risque psychosociaux chez l'enfant », dans J.F. Saucier et L. Houde (dir.), Prévention psychosociale pour l'enfance et l'adolescence, Montréal, Presses de l'Université de Montréal, p. 79-109.

MÉnARD, R. et P. PINARD (1997). Programme d'intervention en négligence, Les Centres jeunesse Mauricie-Bois-Francs. 
MoRISSON, H. (1993). Children of Depressed Parents, Risk, Identification and Intervention, New York, Grune and Stratton.

NICHOLSON, J. et A. BLANCH (1994). "Rehabilitation for parenting roles for people with serious mental illness ", Psychosocial Rehabilitation Journal, vol. 18, p. 109-119.

Nicholson, J., J.L. GelleR, W.H. FisheR et G.L. DION (1993). «State policies and programs that address the needs of mentally ill mothers in the public sector ", Hospital and Community Psychiatry, vol. 44, $n^{\circ} 5$, p. $484-489$.

ORVAschel, H. (1983). «Parental Depression and Child Psychopathology », dans $\mathrm{H}$. Orvaschel (dir.), Childhood Psychopathology and Developpement, New York, Raven Press, p. 53-66.

Ouellette, Fr.-R. et R. B.-DANDURAND (1992). «Parenté et soutien aux familles avec jeunes enfants : entre l'autonomie et la solidarité ", dans G. Pronovost (dir.), Comprendre la famille, Sainte-Foy, Presses de l'Université du Québec, p. 501-514.

PALACIO-Quintin, E. et L. S. ÉTHIER (1993). «La négligence, un phénomène négligé ", Apprentissage et socialisation, vol. 16, p. 153-164.

PREZIOSA, P. et collab. (1992). "Interactive Patterns in the Schizophrenic Family During Hospitalization », Annals of the first Super, Sanita, 28, n 2, p. 315-318.

RÉGIE RÉGIONALE DE LA SANTÉ ET DES SERVICES SOCIAUX DE MONTRÉALCENTRE (1997). Orientations pour la transformation des services de santé mentale - Rapport de consultation, Direction de la programmation. Service des études et de l'évaluation, 22 septembre.

RodNICK, E.H. et M.J. GoldsteIN (1974). «Premorbid Adjustment and the Recovery of Mothering Function in Acute Schizophrenic Women », Journal of Abnormal Psychology, vol. 83, p. 623-628.

SABETTA, Y. (1984). "Étude exploratoire sur le vécu et les besoins de la famille du patient suivi en psychiatrie ", Revue de santé mentale au Québec, vol. 9, n 1, p. 169-173.

SANTÉ QUÉBEC (1987). Et la santé, ça va?, tome 1, Rapport d'enquête Santé Québec, Québec, Les Publications du Québec.

SEIFER, R. (1996). "Les enfants nés de parents atteints de troubles mentaux : état de recherche sur leur évolution et leurs troubles éventuels. " Traduit par Denise Marchand, P.R.I.S.M.E., printemps, vol. $6, n^{\circ} 1$, p. 9-21. 
SHACHNOW, J. (1987). "Preventive Intervention With Children of Hospitalized Psychiatric Patients ", American Journal of Orthopsychiatry, vol. 57, p. 66-77.

TESSIER, L. et M. ClÉMENT (1992). La réadaptation psychosociale en psychiatrie : Défis des années 90, Boucherville, Gaëtan Morin, 238 p.

TOUSIGNANT, M. et V. KovESS (1985). «L'épidémiologie en santé mentale: le cadre conceptuel de l'enquête Santé Québec", Sociologie et société, p. 15-26.

WeIssman, M.M. (1979). "Depressed Parents and Their Children: Implications For Prevention », dans I. Berlin et L. Stone (dir.), Basic Handbook of Child Psychiatry, New York, Basic Books, p. 292-299. 
